\title{
Clinicopathologic Characteristics and Prognosis of Tongue Squamous Cell Carcinoma in Patients with and without a History of Radiation for Nasopharyngeal Carcinoma: A Matched Case-Control Study
}

\author{
Peng Zhang, $\mathrm{MD}^{1,2}$ \\ Li Zhang, PhD',2 \\ Hui Liu, MD ${ }^{1,2}$ \\ Lei Zhao, MD',2 \\ Yong Li, MD',3 \\ Jing-Xian Shen, MD1,4 \\ Oing Liu, MD ${ }^{1,5}$ \\ Meng-Zhong Liu, MD',2 \\ Mian Xi, MD ${ }^{12}$
}

\begin{abstract}
Purpose
Previous studies reported an association between an increased risk of tongue cancer and radiation treatment for nasopharyngeal carcinoma (NPC). This study compared the clinicopathologic characteristics and outcomes of tongue squamous cell carcinoma (TSCC) in patients with and without a history of radiotherapy for NPC.
\end{abstract}

\section{Materials and Methods}

From 1965 to 2009, a total of 73 patients were diagnosed with TSCC with a history of radiotherapy for NPC. The patients were matched in a 1:3 ratio with patients with sporadic TSCC according to age, sex, and year of the TSCC diagnosis. The primary endpoint was the overall survival.

\section{Results}

The median interval from NPC to TSCC was 82 months. The NPC survivors were more likely to be diagnosed with a more advanced T classification, less likely to have lymph node involvement, and more likely to have the tumor located in the dorsum of the tongue than sporadic TSCC. Regarding the histologic characteristics, the NPC survivors were more likely to have a weak lymphocytic host response, low tumor budding, and low risk of a worse pattern of invasion. The sporadic TSCC patients had a better overall survival (hazard ratio, $0.690 ; p=0.033$ ) than the NPC survivors. In competing risks analysis, the cumulative incidence functions for the competing event (documented non-tongue cancer death) were significantly higher in the NPC survivors (Gray's test, $p=0.001$ ).

\section{Conclusion}

TSCC patients with a history of radiotherapy for NPC appear to have particular clinicopathologic features, a poorer survival, and are more likely to die from non-tongue cancer causes than those with sporadic TSCC.
Correspondence: Mian Xi, MD

Department of Radiation Oncology, Sun Yat-sen University Cancer Center, No. 651 Dongfeng East Road, Guangzhou 510060, China

Tel: 86-20-87343385

Fax: 86-20-87343492

Email: ximian@sysucc.org.cn

Received July 16, 2016

Accepted October 3, 2016

Published Online October 11, 2016

${ }^{*}$ Peng Zhang, Li Zhang, and Hui Liu contributed equally to this work.

\section{Key words}

Tongue neoplasms, Second primary cancer, Nasopharyngeal carcinoma, Prognosis, Morbidity

\section{Introduction}

In southern China, nasopharyngeal carcinoma (NPC) is endemic with an incidence ranging from 20 to 30 per 100000
[1]. Advances in technology, particularly intensity-modulated radiotherapy, have brought revolutionary improvements in the management of NPC, with 5-year overall survival (OS) rates exceeding $80 \%$ [2,3]. Unfortunately, the ionizing radiation and chemotherapy regimens of the treat- 
ment are themselves carcinogenic [4]. Several epidemiological studies have reported an increased incidence of second primary cancer in the NPC survival cohort [5-7]. Chen et al. [5] conducted a population-based study including 23,639 NPC patients and reported the oral cavity and pharynx to be the most common sites of a second primary cancer after radiation for NPC. Previous studies have reported an association between an increased risk of tongue cancer and radiation for NPC [6,8]. Most of this excess risk can be attributed to radiation of the oral cavity [8]. A previous study reported the tongue to be the most common site of second malignancies after NPC [9]. On the other hand, the clinicopathologic characteristics and outcomes of tongue cancer arising in NPC patients have not been explored in detail.

This study analyzed a large series of consecutive patients diagnosed with tongue squamous cell carcinoma (TSCC) at a single institution and identified patients with a prior history of radiotherapy for NPC. The clinicopathologic characteristics and survival were compared with a matched control group of patients with sporadic TSCC.

\section{Materials and Methods}

\section{Patient and disease characteristics}

The inclusion criteria were (1) a prior history of radiotherapy for NPC, (2) histological confirmation of TSCC, and (3) a latency period of at least 3 years between the end of radiotherapy and the diagnosis of TSCC. The criteria were adapted from those described by Cahan et al. [10] and Arlen et al. [11], who applied them to radiation-induced sarcomas. Such cases were excluded from the study sample because of the different treatment principles of tongue-base malignancies owing to the anatomical position.

From January 1965 to December 2009, 56,127 patients with NPC received radiotherapy at the Sun Yat-sen University Cancer Center. Of these, a total of 73 patients fulfilled the inclusion criteria. A total of 2,720 consecutive patients were diagnosed with tongue cancer with no history of concomitant or prior malignancy in the authors' institution. For each NPC survivor, three matched patients (219 patients) with sporadic tongue cancer were selected. The two groups were matched according to the most relevant variables: sex, age at tongue cancer diagnosis (within 5 years), and year of the tongue cancer diagnosis (within 3 years). During the matching process, the investigators were blinded to the clinical outcomes of the TSCC. All patients underwent a regular follow-up at the authors' hospital. The date of the last follow-up was September 2015. The institutional ethics commit- tees of Sun Yat-sen University Cancer Center approved this study in accordance with the Declaration of Helsinki and written informed consent was obtained from all surviving patients or family members of the dead patients.

The radiation techniques for NPC are described in detail elsewhere $[12,13]$. The majority of patients were treated with conventional radiotherapy and the daily fraction was $2 \mathrm{~Gy}$ delivered using Cobalt-60 or 6-MV X-rays. Briefly, the twodimensional radiation technique consisted of two phases. In the first phase, $50 \mathrm{~Gy}$ was delivered to the nasopharyngeal region with two lateral facial-cervical fields (Fig. 1A). In the second phase, a total dose of 16-26 Gy was delivered to the primary lesions in a smaller preauricular field (Fig. 1B). The anterior and posterior split fields were used in the neck region. The radiation dose to the cervical region was 50-54 Gy for N0 stage and 60-66 Gy for any positive lymph nodes.

The clinicopathologic characteristics of the TSCC patients and treatment modalities for TSCC were identified, including radiotherapy, surgery, and multimodality therapy. All patients diagnosed with TSCC were restaged according to the seventh edition of the American Joint Committee on the Cancer TNM staging system. Hematoxylin and eosin (H\&E)stained slides have been available at the authors' institution since 1997. Of the 141 patients, including the NPC survivors and the matched group from 1997 to 2009, 62 underwent biopsies at other hospitals and received treatment without surgery. Therefore, the H\&E slides of only 79 patients were available, which included 22 NPC survivors and 57 sporadic TSCC patients. All slides were re-evaluated independently by optical microscopy by experienced pathologists, and then jointly for consensus. The investigators were blinded to the clinical data at the time of the evaluation. The H\&E-stained slides were assessed with respect to the presence of the worst pattern of invasion (WPOI), tumor budding, lymphocytic host response (LHR), vascular invasion (VI) or perineural invasion (PNI), and depth of tumor invasion.

The LHR pattern was classified as a three-tiered variable (strong, intermediate, or weak) based on the presence of lymphoid nodules. Tumors with a strong LHR were identified as showing the presence of at least one interface lymphoid nodule per $\times 4$ objective lens on optical microscopy. Intermediate LHR was assigned when tumors with lymphoid responses were below this threshold but with one or more lymphoid nodules. Weak LHR was defined as a limited response that lacked any lymphoid nodules [14]. High-risk WPOI was defined as small tumor islands fewer than 15 cells and satellite tumors, which were located $\geq 1 \mathrm{~mm}$ away from the main tumor or the nearest satellite. The presence of PNI and limited or no LHR were also considered to be high-risk WPOI. Low-risk WPOI was assigned when the tumors showed pushing borders, large cohesive invasion, or fingerlike growth [15]. Tumor budding was defined as the presence 


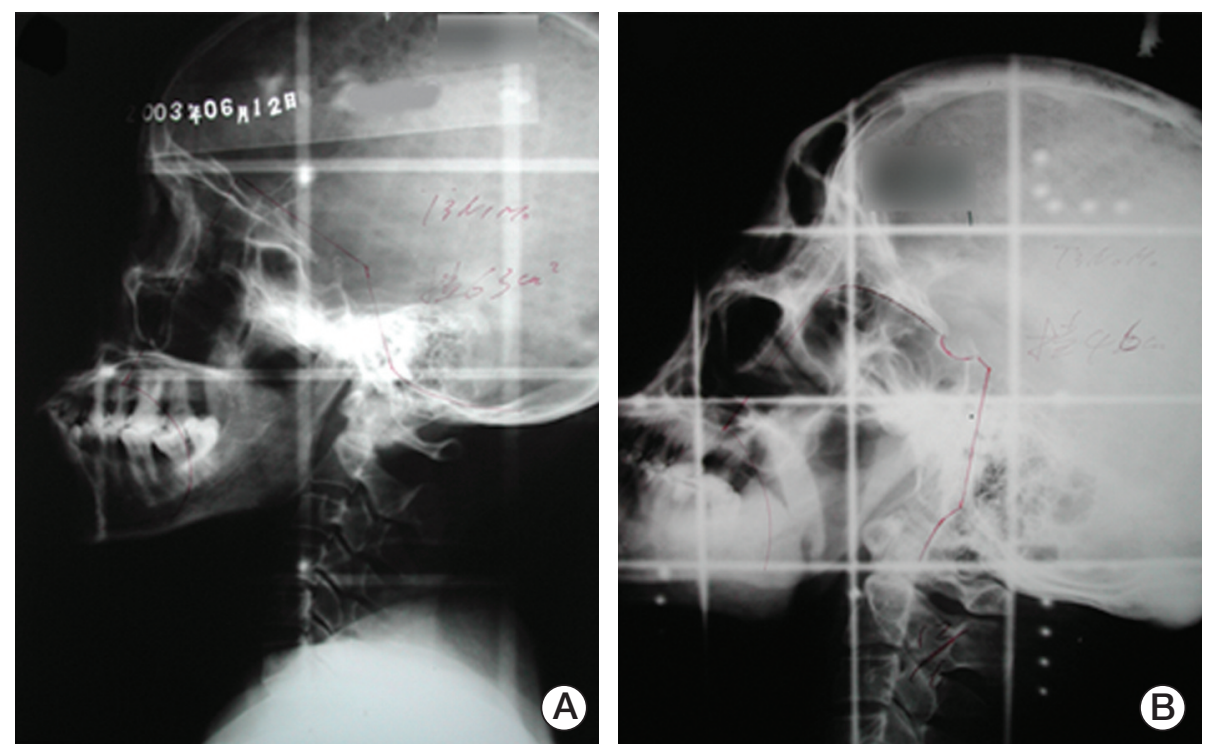

Fig. 1. The radiation field for nasopharyngeal carcinoma with conventional two-dimensional radiation techniques. (A) Phase I. (B) Phase II.

of a single cancer cell or a cluster of fewer than five cancer cells in the invasive frontal region [16]. The slides were initially scanned at a $\times 4$ magnification to select the areas with the highest density of tumor budding. Tumor budding in the selected areas was then counted at a $\times 20$ magnification; the highest count per slide was identified as the number of tumor buds. Tumor budding $\geq 5$ was considered to be high risk. The presence of a large PNI and little or no LHR were also considered to be high risk [17].

\section{Statistical analysis}

A Pearson chi-square test or Fisher exact test was used to assess the associations between the patient's characteristics and cohort membership (NPC survivors vs. matched cohort). The OS was the primary endpoint in this study and was calculated from the time of the TSCC diagnosis until death or the last follow-up. The disease-free survival (DFS) was defined as the time from the TSCC diagnosis to the first evidence of any treatment failure or death or the last follow-up. Survival analysis was performed using the Kaplan-Meier method; the log-rank test was used to evaluate the statistical significance of the differences. To assess the unadjusted impact of a history of radiotherapy for NPC, competing risks analysis was applied to compare the different types of deaths between the NPC survivors and matched cohort. The Gray's test was applied to compare the cumulative incidence functions estimated in competing risks analysis [18]. A multivariate Cox proportional hazards model was fitted to assess the independent prognostic significance of the clinical and pathologic characteristics and calculate the hazard ratios. A p-value of $<0.05$ was considered significant.

\section{Results}

\section{NPC characteristics and treatment in the survivor cohort}

The crude incidence of TSCC after receiving radiotherapy for NPC was estimated to be approximately $0.13 \%$. Table 1 lists the patient demographics for the NPC survivor cohort at the time of the NPC diagnosis. The median age at the NPC and TSCC diagnosis was 43 years (range, 23 to 66 years) and 52 years (range, 35 to 72 years), respectively. The majority of NPC cases were diagnosed with stage II and III (95.9\%) cancers. The median cumulative radiation dose to the nasopharyngeal and neck region was $68.0 \mathrm{~Gy}$ (range, 57.3 to $88.0 \mathrm{~Gy}$ ) and 52.0 Gy (range, 42.0 to $78.0 \mathrm{~Gy}$ ), respectively. Fifteen patients $(20.5 \%)$ received chemotherapy with alkylating agents as the treatment. The median interval from NPC to TSCC diagnosis was 82 months (range, 37 to 308 months). The second tongue cancer occurred within 5 years since the end of radiotherapy for NPC in $37.0 \%$ of patients, within 10 years in $68.5 \%$ of patients, and within 15 years in $91.8 \%$ of patients. 
Table 1. Demographic and clinical characteristics of NPC in the survivor cohort

\begin{tabular}{|c|c|}
\hline Characteristic & No. $(\%)(n=73)$ \\
\hline \multicolumn{2}{|l|}{ Year of NPC diagnosis } \\
\hline Before 1990 & $34(46.6)$ \\
\hline 1990-1999 & $30(41.1)$ \\
\hline$\geq 2000$ & $9(12.3)$ \\
\hline \multicolumn{2}{|l|}{ Sex } \\
\hline Male & $54(74.0)$ \\
\hline Female & $19(26.0)$ \\
\hline \multicolumn{2}{|l|}{ NPC stage at diagnosis } \\
\hline I & $2(2.7)$ \\
\hline II & $40(54.8)$ \\
\hline III & $30(41.1)$ \\
\hline IV & $1(1.4)$ \\
\hline \multicolumn{2}{|l|}{ Radiation machine } \\
\hline Orthovoltage $\mathrm{X}$-rays & $5(6.9)$ \\
\hline Cobalt-60 & $51(69.9)$ \\
\hline Megavoltage X-rays & $17(23.2)$ \\
\hline \multicolumn{2}{|l|}{ Radiation technique } \\
\hline Conventional radiotherapy & $70(95.9)$ \\
\hline 3DCRT/IMRT & $3(4.1)$ \\
\hline \multicolumn{2}{|l|}{ Radiation course } \\
\hline Split & $35(47.9)$ \\
\hline Continuous & $38(52.1)$ \\
\hline \multicolumn{2}{|l|}{ Alkylating agents chemotherapy } \\
\hline Yes & $15(20.5)$ \\
\hline No & $58(79.5)$ \\
\hline \multicolumn{2}{|l|}{ Local or regional recurrence } \\
\hline Yes & $9(12.3)$ \\
\hline No & $64(87.7)$ \\
\hline Radiation dose, median (range, Gy) & $68.0(57.3-88.0)$ \\
\hline Age at NPC diagnosis, median (range, yr) & $43(23-66)$ \\
\hline Age at TSCC diagnosis, median (range, yr) & $52(35-72)$ \\
\hline $\begin{array}{l}\text { Latency from NPC to TSCC, median } \\
\text { (range, mo) }\end{array}$ & $82.0(37-308)$ \\
\hline
\end{tabular}

NPC, nasopharyngeal carcinoma; 3DCRT, 3-dimensional conformal radiotherapy; IMRT, intensity-modulated radiotherapy; TSCC, tongue squamous cell carcinoma.

\section{TSCC characteristics and treatment: NPC survivors in comparison with the matched group}

The median age of the sporadic TSCC cohort $(2,720$ patients: 1,796 male [66.0\%] and 924 female [34.0\%]) was 52 years (range, 20 to 94 years; mean, 51.7 years). The age of onset of TSCC $(\mathrm{p}=0.433)$ and sex distribution $(\mathrm{p}=0.169)$ were similar between the NPC survivors and the sporadic TSCC cohort.

As shown in Table 2, the NPC survivors and matched cohort showed a similar TNM stage distribution of TSCC. On the other hand, the NPC survivors were more likely to have a more advanced $\mathrm{T}$ classification (T4 classification, $17.8 \%$ vs. $2.7 \%$ ) and less likely to have lymph node involvement $(15.1 \%$ vs. $24.2 \%$ ) than the matched cohort. The tumor location in the NPC survivor cohort was more likely to be located in the dorsum of the tongue than that observed in the matched cohort (35.6\% vs. 9.1\%). In addition, the NPC survivors were more likely to have a family history of cancer $(16.4 \%$ vs. $8.7 \%)$.

The treatment modality used showed significant intergroup differences with $67.1 \%$ of the NPC survivors receiving surgery alone or multimodality therapy versus $89.0 \%$ in the matched group $(p<0.001)$. A neck dissection was performed in $21.9 \%$ of NPC survivors compared to $74.9 \%$ in the matched group $(p<0.001)$. The rates of chemotherapy and radiotherapy did not differ significantly between the two groups.

\section{Histological comparison}

A total of 79 patients were included for a histological comparison (Fig. 2). As shown in Table 2, there was no significant difference between the NPC survivors and matched group with respect to VI and PNI. A weak LHR was evident in the 18 NPC survivors (81.8\%), whereas only 25 sporadic TSCC patients $(43.9 \%)$ had a weak LHR ( $\mathrm{p}=0.003)$. In addition, a significantly higher number $(75.4 \%)$ of sporadic TSCC patients had a high intensity of tumor budding $(\geq 5)$, whereas only $45.5 \%$ of NPC survivors had this risk $(\mathrm{p}=0.016)$. The high risk of WPOI was significantly higher in the sporadic TSCC patients $(68.4 \%)$ than in the NPC survivors (40.9\%) $(\mathrm{p}=0.039)$.

\section{Follow-up and survival analysis}

The median follow-up time of the NPC survivors and the matched group after the TSCC diagnosis who were alive was 90.0 months and 114.0 months, respectively. The OS was significantly poorer in the NPC survivor group than the matched group (5-year OS rates, $44.0 \%$ vs. $63.6 \%$; $\mathrm{p}=0.006$ ) (Fig. 3A). A significant difference in the DFS was observed between the 2 groups (5-year DFS, $39.0 \%$ vs. $53.4 \%$; $\mathrm{p}=0.026$ ) (Fig. 3B). In competing risks analysis, the cumulative incidence functions for the tongue cancer events were similar in the two groups (Gray's test, $\mathrm{p}=0.331$ ), but the rates of the competing event (documented non-tongue cancer death) were significantly higher in the NPC survivors (Gray's test, $\mathrm{p}=0.001$ ).

During the follow-up, four patients (5.5\%) in the NPC survivor group developed a third primary cancer, namely diffuse large B cell lymphoma, left breast cancer, thyroid cancer, and basal-cell epithelioma in the neck, while five patients 
Table 2. Characteristics and treatment in NPC survivors and matched patients with sporadic TSCC

\begin{tabular}{|c|c|c|c|}
\hline Characteristic & NPC survivors & Sporadic TSCC & p-value \\
\hline \multicolumn{4}{|l|}{ Stage } \\
\hline I & $26(35.6)$ & $70(32.0)$ & 0.153 \\
\hline II & $20(27.4)$ & $87(39.7)$ & \\
\hline III & $14(19.2)$ & $40(18.3)$ & \\
\hline IVa & $13(17.8)$ & $22(10.0)$ & \\
\hline \multicolumn{4}{|l|}{$T$ classification } \\
\hline $\mathrm{T} 1$ & $27(37.0)$ & $85(38.8)$ & $<0.001$ \\
\hline $\mathrm{T} 2$ & $25(34.2)$ & $114(52.1)$ & \\
\hline T3 & $8(11.0)$ & $14(6.4)$ & \\
\hline $\mathrm{T} 4$ & $13(17.8)$ & $6(2.7)$ & \\
\hline \multicolumn{4}{|l|}{$\mathrm{N}$ classification } \\
\hline No & $62(84.9)$ & $166(75.8)$ & 0.031 \\
\hline N1 & $11(15.1)$ & $37(16.9)$ & \\
\hline $\mathrm{N} 2$ & 0 & $16(7.3)$ & \\
\hline \multicolumn{4}{|l|}{ Site of primary tumor } \\
\hline Lateral & $43(58.9)$ & $149(68.0)$ & $<0.001$ \\
\hline Ventral & $4(5.5)$ & $50(22.8)$ & \\
\hline Dorsal & $26(35.6)$ & $20(9.1)$ & \\
\hline \multicolumn{4}{|l|}{ Pathological grading } \\
\hline I & $58(79.5)$ & $147(67.1)$ & 0.019 \\
\hline II & $12(16.4)$ & $42(19.2)$ & \\
\hline III & $2(2.7)$ & $4(1.8)$ & \\
\hline Unknown & $1(1.4)$ & $26(11.9)$ & \\
\hline \multicolumn{4}{|l|}{ Family history of cancer } \\
\hline Yes & $12(16.4)$ & $19(8.7)$ & 0.062 \\
\hline No & $61(83.6)$ & $200(91.3)$ & \\
\hline \multicolumn{4}{|l|}{ Alcohol consumption } \\
\hline Yes & $10(15.9)$ & $66(30.1)$ & 0.006 \\
\hline No & $63(84.1)$ & $153(69.9)$ & \\
\hline \multicolumn{4}{|l|}{ Smoking } \\
\hline Yes & $29(39.7)$ & $107(48.9)$ & 0.176 \\
\hline No & $44(60.3)$ & $112(51.1)$ & \\
\hline \multicolumn{4}{|l|}{ Treatment } \\
\hline Surgery alone & $33(45.2)$ & $125(57.0)$ & $<0.001$ \\
\hline Chemotherapy alone & $14(19.2)$ & $7(3.2)$ & \\
\hline Radiotherapy alone & $10(13.7)$ & $17(7.8)$ & \\
\hline Multimodality therapy & $16(21.9)$ & $70(32.0)$ & \\
\hline \multicolumn{4}{|l|}{ Neck dissection } \\
\hline Surgery with neck dissection & $16(21.9)$ & $164(74.9)$ & $<0.001$ \\
\hline Surgery without neck dissection & $27(37.0)$ & $23(10.5)$ & \\
\hline No surgery & $30(41.1)$ & $32(14.6)$ & \\
\hline \multicolumn{4}{|l|}{ Any chemotherapy } \\
\hline Yes & $29(39.7)$ & $63(28.8)$ & 0.081 \\
\hline No & $44(60.3)$ & $156(71.2)$ & \\
\hline \multicolumn{4}{|l|}{ Any radiotherapy } \\
\hline Yes & $15(20.5)$ & $49(22.4)$ & 0.744 \\
\hline No & $58(79.5)$ & $170(77.6)$ & \\
\hline \multicolumn{4}{|l|}{ Vascular invasion } \\
\hline Present & $2(9.1)$ & $3(5.3)$ & 0.614 \\
\hline Absent & $20(90.9)$ & $54(94.7)$ & \\
\hline
\end{tabular}


Table 2. Continued

\begin{tabular}{|c|c|c|c|}
\hline Characteristic & NPC survivors & Sporadic TSCC & p-value \\
\hline \multicolumn{4}{|l|}{ Perineural invasion } \\
\hline Present & $2(9.1)$ & $1(1.8)$ & 0.186 \\
\hline Absent & $20(90.9)$ & $56(98.2)$ & \\
\hline \multicolumn{4}{|l|}{ LHR } \\
\hline Strong/Intermediate & $4(18.2)$ & $32(56.1)$ & 0.003 \\
\hline Weak & $18(81.8)$ & $25(43.9)$ & \\
\hline \multicolumn{4}{|l|}{ Tumor budding } \\
\hline $\operatorname{High}(\geq 5)$ & $10(45.5)$ & $43(75.4)$ & 0.016 \\
\hline Low $(<5)$ & $12(54.5)$ & $14(24.6)$ & \\
\hline \multicolumn{4}{|l|}{ Worst pattern of invasion } \\
\hline High & $9(40.9)$ & $39(68.4)$ & 0.039 \\
\hline Low & $13(59.1)$ & $18(31.6)$ & \\
\hline
\end{tabular}

Values are presented as number (\%). NPC, nasopharyngeal carcinoma; TSCC, tongue squamous cell carcinoma; LHR, lymphocytic host response.
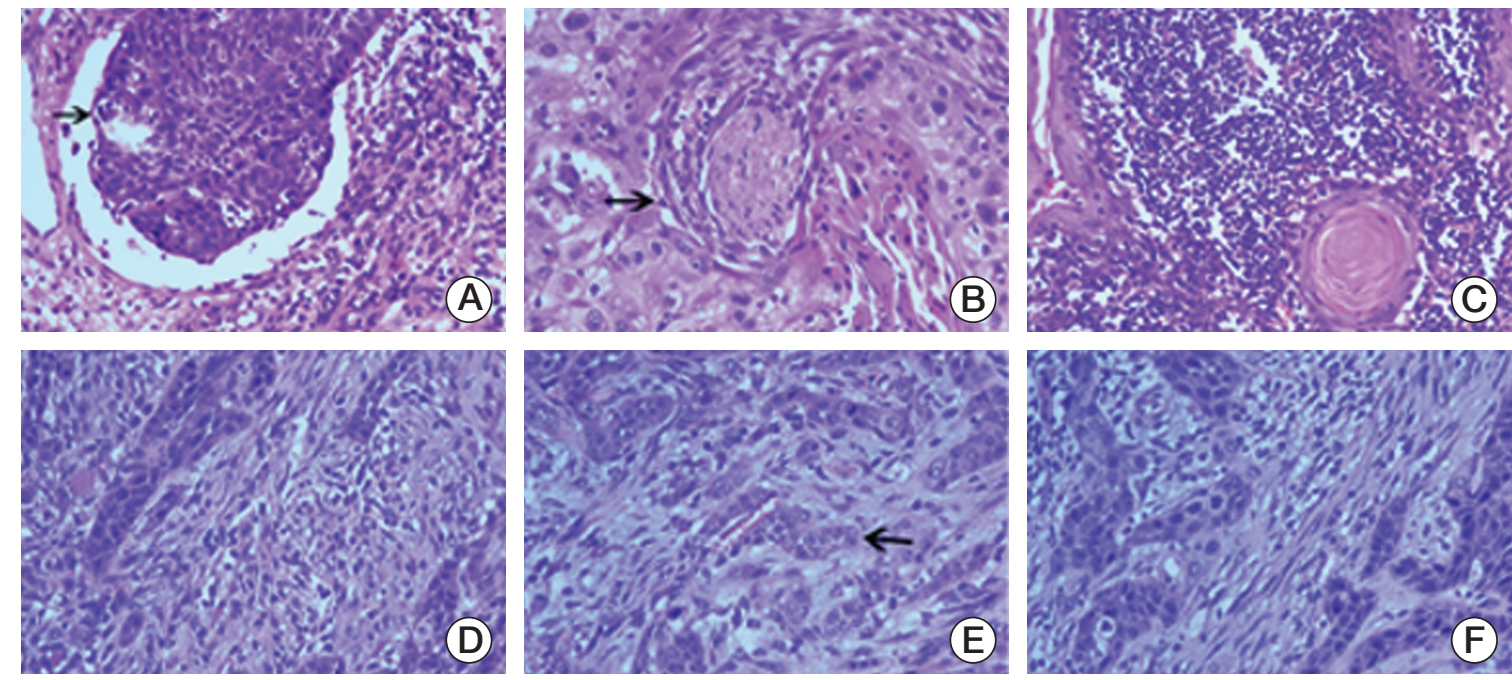

Fig. 2. Histologic features of tongue squamous cell carcinoma, presence of vascular invasion (arrow) (A), presence of perineural invasion (arrow) (B), strong lymphocytic host response (C), weak lymphocytic host response (D), presence of tumor budding (arrow) (E), and presence of high risk of worst pattern of invasion (F) (H\&E staining, $\times 200)$.

$(2.3 \%)$ in the matched group developed a second primary cancer.

Table 3 lists the results of univariate analysis for the OS and DFS. The statistically significant clinical factors in univariate analysis $(p<0.10)$ were analyzed by multivariate analysis (Table 4). The multivariate Cox proportional hazards regression model yielded a hazard ratio (HR) of 0.690 ( $95 \%$ confidence interval $[\mathrm{CI}], 0.491$ to $0.971 ; \mathrm{p}=0.033$ ) for the matched group compared to the NPC survivor cohort, with the OS as the endpoint. Independent of the NPC history, the risk of death due to any cause was also greater in male patients, patients with a more advanced TNM stage, and those who did not receive surgery or multimodality treatment. On the other hand, the NPC history was not an independent prognostic factor for the DFS (HR, 0.768; 95\% CI, 0.554 to $1.063 ; p=0.112)$. The TNM stage $(p<0.001)$ and treatment modality $(\mathrm{p}=0.025)$ appeared to have independent effects on the DFS. 
A

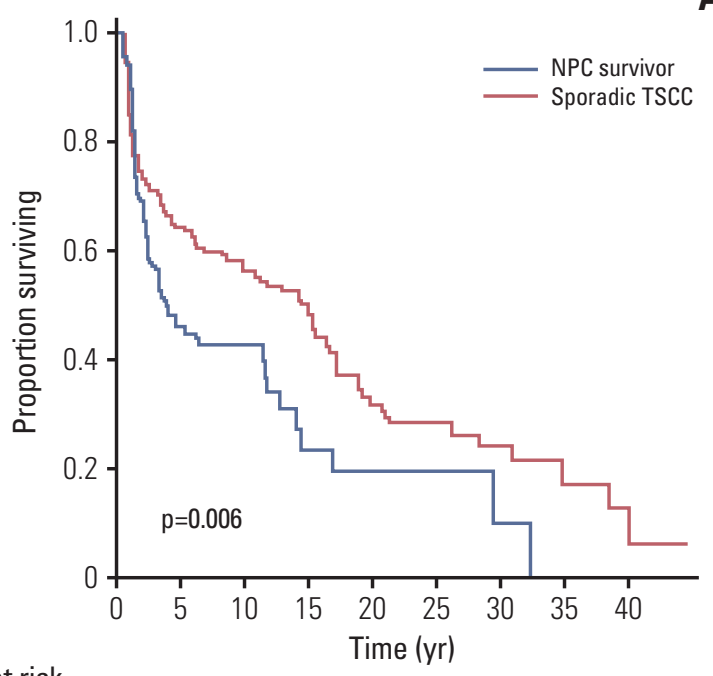

No. at risk

$\begin{array}{llllllllll}\text { NPC survivor } & 72 & 28 & 17 & 6 & 3 & 2 & 1 & 0 & 0\end{array}$

$\begin{array}{llllllllll}\text { Sporadic TC } & 219 & 122 & 74 & 43 & 21 & 15 & 10 & 4 & 2\end{array}$

Fig. 3. (A) Overall survival for nasopharyngeal carcinoma (NPC) survivors and matched patients with sporadic tongue squamous cell carcinoma (TSCC) ( $\mathrm{p}=0.006)$. (B) Disease-free survival for NPC survivors and matched patients with sporadic $\operatorname{TSCC}(\mathrm{p}=0.026)$. TC, tongue cancer.

\section{Discussion}

The standardized incidence ratios of tongue cancer were significantly higher in NPC patients, particularly in NPC endemic areas [7]. In this study, 73 TSCC patients, who had a history of radiotherapy for NPC, were compared with a matched cohort of 219 sporadic TSCC cases. To the best of the authors' knowledge, this is the largest study to compare TSCC patients with a history of radiotherapy for NPC and sporadic TSCC cases. This study showed that the clinicopathologic characteristics and outcomes were significantly different between the two groups.

Up to now, there is no definite conclusion regarding the pathogenesis of radiation-related second primary tumors. Teo et al. [8] suggested that the increasing incidence of tongue cancer in NPC patients was more likely associated with a history of oral cavity radiotherapy than the sharing of common risk factors or mucosal field-cancerization. Wolden et al. [19] reported the long-term monitoring results of 694 children and teenagers after treatment for Hodgkin's disease and reported that $93 \%$ of second solid malignancies developed in regions that had received at least $35 \mathrm{~Gy}$. Regarding the radiation field of conventional radiotherapy in NPC, the anterior half of the tongue was shielded routinely. On the other hand, the posterior tongue dorsum and tongue base were inevitably included in the medium- and high-dose region of the radiation field. As reported by Qin et al. [20] the mean radiation dose to the tongue tip, tongue body, tongue base, and entire tongue was 11.2 Gy, 34.1 Gy, 57.0 Gy, and $43.1 \mathrm{~Gy}$, respectively, using a two-dimensional radiation technique. In addition, the distribution of the tongue cancer location between the NPC survivor group and sporadic TSCC cohort is significantly different. TSCC in NPC survivors is more likely to be located in the dorsum of the tongue but less likely in the ventral area. Therefore, the purported correlation between the radiation dose and the location of second primary tumor was confirmed.

Compared to sporadic TSCC, TSCC in NPC survivors was more likely to have a more advanced $\mathrm{T}$ classification, but less likely to have lymph node involvement. The relatively higher percentage of T4 classification in NPC survivors may be because the tumor was more likely to be located in the dorsum of the tongue than the sporadic TSCC cohort (35.6\% vs. $9.1 \%, \mathrm{p}<0.001)$. The lesions located in the dorsum may more easily involve the adjacent structures (e.g., deep muscle of the tongue) and were almost imperceptible in the early stage. On the other hand, TSCC in the NPC survivors was less likely to have lymph node involvement, which may be due to two reasons: a history of radiation to neck region causes the lymphatic atresia and altered the lymphatic drainage; and the significantly higher percentage of neck dissection in 
Table 3. Univariate analysis of OS and DFS in relation to clinical characteristics

\begin{tabular}{|c|c|c|c|c|c|}
\hline \multirow{2}{*}{ Variable } & \multirow{2}{*}{ No. $(\%)$} & \multicolumn{2}{|c|}{ OS } & \multicolumn{2}{|c|}{ DFS } \\
\hline & & 5-Year OS & p-value & 5-Year DFS & p-value \\
\hline \multicolumn{6}{|l|}{ Group } \\
\hline NPC survivor & $73(25.0)$ & 44.0 & 0.006 & 39.0 & 0.026 \\
\hline Sporadic TSCC & $219(75.0)$ & 63.6 & & 53.4 & \\
\hline \multicolumn{6}{|l|}{ Age (yr) } \\
\hline$\leq 52$ & $154(52.7)$ & 62.0 & 0.159 & 53.5 & 0.139 \\
\hline$>52$ & $138(47.3)$ & 57.5 & & 46.4 & \\
\hline \multicolumn{6}{|l|}{ Sex } \\
\hline Male & $198(67.8)$ & 53.5 & 0.007 & 45.7 & 0.029 \\
\hline Female & $94(32.2)$ & 67.2 & & 56.8 & \\
\hline \multicolumn{6}{|l|}{ Family history of cancer } \\
\hline Yes & $31(10.6)$ & 52.9 & 0.833 & 43.8 & 0.624 \\
\hline No & $261(89.4)$ & 58.5 & & 50.4 & \\
\hline \multicolumn{6}{|l|}{ Alcohol consumption } \\
\hline Yes & $76(26.0)$ & 51.7 & 0.534 & 47.7 & 0.974 \\
\hline No & $216(74.0)$ & 60.3 & & 50.4 & \\
\hline \multicolumn{6}{|l|}{ Smoking } \\
\hline Yes & $136(46.6)$ & 56.0 & 0.331 & 45.8 & 0.356 \\
\hline No & $156(53.4)$ & 61.8 & & 54.0 & \\
\hline \multicolumn{6}{|l|}{ TNM stage } \\
\hline I-II & $203(69.5)$ & 70.5 & $<0.001$ & 60.6 & $<0.001$ \\
\hline III-IV & $89(30.5)$ & 31.0 & & 26.4 & \\
\hline \multicolumn{6}{|l|}{ Pathological grading } \\
\hline I-II & $259(88.7)$ & 59.3 & 0.983 & 50.1 & 0.456 \\
\hline III/Unknown & $33(11.3)$ & 57.1 & & 50.8 & \\
\hline \multicolumn{6}{|l|}{ Tumor location } \\
\hline Lateral & $192(65.8)$ & 60.9 & 0.160 & 52.3 & 0.148 \\
\hline Ventral & $54(18.5)$ & 57.6 & & 50.5 & \\
\hline Dorsal & $46(15.8)$ & 49.8 & & 35.8 & \\
\hline \multicolumn{6}{|l|}{ Treatment modality } \\
\hline Surgery alone & $158(54.1)$ & 70.8 & $<0.001$ & 61.5 & $<0.001$ \\
\hline Chemotherapy alone & $21(7.2)$ & 4.8 & & 0.0 & \\
\hline Radiotherapy alone & $27(9.2)$ & 58.3 & & 43.3 & \\
\hline Multimodality therapy & $86(29.5)$ & 49.9 & & 40.0 & \\
\hline \multicolumn{6}{|l|}{ Neck dissection } \\
\hline Surgery with neck dissection & $180(61.6)$ & 68.3 & $<0.001$ & 60.0 & $<0.001$ \\
\hline Surgery without neck dissection & $50(17.1)$ & 55.3 & & 44.8 & \\
\hline No surgery & $62(21.3)$ & 33.0 & & 27.0 & \\
\hline
\end{tabular}

OS, overall survival; DFS, disease-free survival; NPC, nasopharyngeal carcinoma; TSCC, tongue squamous cell carcinoma.

the sporadic TSCC cohort (74.9\% vs. $21.9 \%$, p $<0.001)$ leads to a relatively higher percentage of positive lymph node detection.

A detailed histopathologic review showed that TSCC in the NPC survivors was more likely to have weak LHR, lowrisk WPOI, and low tumor budding. Previous studies reported that the increased risks of second primary malignancies could be attributed to the compromised immune function in cancer survivors. The strong LHR suggested that an adaptive cytotoxic T-cell immune response was concentrated significantly at the interface of the tumors and the strong LHR has a protective effect in oral cancer patients [21]. Therefore, the weak LHR in NPC survivors indicated that these patients may have an impaired immune surveillance and adaptive immunity. Tumor budding represents two additional aggressive and malignant features (active inva- 
Table 4. Multivariate analysis of OS and DFS in relation to clinical characteristics

\begin{tabular}{llcrr} 
Endpoint & \multicolumn{1}{c}{ Characteristic } & HR & 95\% CI for HR & p-value \\
OS & History of NPC & 0.690 & $0.491-0.971$ & 0.033 \\
& Sex & 0.686 & $0.476-0.988$ & 0.043 \\
& TNM stage & 2.146 & $1.553-2.964$ & $<0.001$ \\
\hline \\
DFS & Treatment modality & 1.158 & $1.029-1.303$ & 0.015 \\
& Neck dissection & 1.054 & $0.807-1.376$ & 0.701 \\
& History of NPC & 0.768 & $0.554-1.063$ & 0.112 \\
& Sex & 0.786 & $0.548-1.078$ & 0.127 \\
& TNM stage & 1.766 & $1.285-2.426$ & $<0.001$ \\
& Treatment modality & 1.137 & $1.016-1.271$ & 0.025 \\
\hline
\end{tabular}

OS, overall survival; DFS, disease-free survival; HR, hazard ratio; CI, confidence interval; NPC, nasopharyngeal carcinoma.

sion and cellular discohesion). The presence of high intensity tumor budding was associated with a poorer prognosis, epithelialmesenchymal transition, and cervical lymph node metastasis in TSCC $[17,22]$. In this study, the sporadic TSCC patients were more likely to have lymph node involvement than TSCC in the NPC survivors, which validated this pathological feature. The high-risk WPOI, which is marked by small tumor islands and satellite tumor, was also confirmed as an independent predictor for a poor prognosis in TSCC [15]. Overall, TSCC in NPC survivors appear to have less aggressive histologic features (low tumor budding and lowrisk WPOI) but impaired immune surveillance (weak LHR). The distinctive histological features in radiation-associated TSCC patients should be validated in a further study.

There has been increasing concern as to whether a prior history of cancer could impact the prognosis and therapeutic management in patients with head and neck cancer [23]. A major finding of this analysis was the negative prognostic impact of a history of radiotherapy for NPC on the survival of patients subsequently diagnosed with TSCC, which has never been examined previously. On the other hand, death as a result of tongue cancer was similar in the NPC survivors and sporadic TSCC cohort. This is because NPC survivors were more likely to die from non-tongue cancer causes. The accumulation of genetic damage due to a history of radiation and the differences in the immune status may help explain this situation [24,25]. In addition, the prognosis of TSCC in NPC survivors could be negatively influenced by the excess mortality due to NPC and severe late complications. Similar results were obtained in other second cancers, such as breast cancer, among patients with a history of radiation for Hodgkin's lymphoma [26]. In particular, the cumulative incidence functions for the tongue cancer events were similar in the two groups (Gray's test, $\mathrm{p}=0.331$ ). Several possible interpretations can be made. First, the potential survival advantage of less aggressive histologic features in TSCC of NPC survivors (low tumor budding and low-risk WPOI) may be counteracted by the impaired immune surveillance (weak LHR). Second, the TSCC in NPC survivors were more likely to be limited to local disease without lymphatic nodal metastases, which was correlated with better local control and longer survival. Third, previous studies indicated that TSCC in the NPC survivors and sporadic TSCC might have different carcinogenesis [8]. The sporadic TSCC patients were more likely to be accompanied by mucosal field-cancerization, which resulted in a difficulty of loco-regional control.

The main limitations of this study were its retrospective nature and single-institutional data design. Although deaths as a result of tongue cancer could be identified in this study, there was limited information on the specific causes of noncancer deaths because of the unexplained loss to follow-up. In addition, this study spanned more than 40 years, during which radiotherapy technology for NPC has evolved markedly. The majority of NPC patients in this study received conventional radiotherapy owing to the relatively short follow-up time for the patients who received modern conformal radiotherapy. The relevance of the long-term effects of outdated treatments to patients receiving modern therapy is still questionable. The main strength of this analysis was its very large sample size of radiation-associated TSCC in the NPC survivor cohort. Considering the rarity of radiation-associated $\mathrm{TSCC}$, the generalizability of these results will require further validation in multi-centric studies. 


\section{Conclusion}

Compared to sporadic TSCC, TSCC s with a history of NPC radiation have specific clinicopathologic features. These results also indicated that the history of radiation for NPC was a poor prognostic factor. TSCC patients with a history of radiotherapy for NPC are more likely to die from nontongue cancer causes than those with sporadic TSCC. These results may impact cancer-screening strategies in patients with NPC, and those with a high risk should be targeted for more intensive surveillance.

\section{Conflicts of Interest}

Conflict of interest relevant to this article was not reported.

\section{Acknowledgments}

This research was supported by the grants from the SciTech Project Foundation of Guangdong Province (2012B061700083), and the National Natural Science Foundation of China (81201748).

\section{References}

1. Wei WI, Sham JS. Nasopharyngeal carcinoma. Lancet. 2005; 365:2041-54.

2. Lee AW, Sze WM, Au JS, Leung SF, Leung TW, Chua DT, et al. Treatment results for nasopharyngeal carcinoma in the modern era: the Hong Kong experience. Int J Radiat Oncol Biol Phys. 2005;61:1107-16.

3. Tham IW, Hee SW, Yeo RM, Salleh PB, Lee J, Tan TW, et al. Treatment of nasopharyngeal carcinoma using intensity-modulated radiotherapy-the national cancer centre singapore experience. Int J Radiat Oncol Biol Phys. 2009;75:1481-6.

4. Travis LB, Demark Wahnefried W, Allan JM, Wood ME, Ng AK. Aetiology, genetics and prevention of secondary neoplasms in adult cancer survivors. Nat Rev Clin Oncol. 2013;10: 289-301.

5. Chen MC, Feng IJ, Lu CH, Chen CC, Lin JT, Huang SH, et al. The incidence and risk of second primary cancers in patients with nasopharyngeal carcinoma: a population-based study in Taiwan over a 25-year period (1979-2003). Ann Oncol. 2008;19: 1180-6.

6. Scelo G, Boffetta P, Corbex M, Chia KS, Hemminki K, Friis S, et al. Second primary cancers in patients with nasopharyngeal carcinoma: a pooled analysis of 13 cancer registries. Cancer Causes Control. 2007;18:269-78.

7. Kong L, Lu JJ, Hu C, Guo X, Wu Y, Zhang Y. The risk of second primary tumors in patients with nasopharyngeal carcinoma after definitive radiotherapy. Cancer. 2006;107:1287-93.

8. Teo PM, Chan AT, Leung SF, Chau RM, Yu PK, King WW, et al. Increased incidence of tongue cancer after primary radiotherapy for nasopharyngeal carcinoma: the possibility of radiation carcinogenesis. Eur J Cancer. 1999;35:219-25.

9. Xi M, Liu SL, Zhao L, Shen JX, Zhang L, Zhang P, et al. Prognostic factors and survival in patients with radiation-related second malignant neoplasms following radiotherapy for nasopharyngeal carcinoma. PLoS One. 2013;8:e84586.

10. Cahan WG, Woodard HQ, Higinbotham NL, Stewart FW,
Coley BL. Sarcoma arising in irradiated bone: report of eleven cases: 1948. Cancer. 1998;82:8-34.

11. Arlen M, Higinbotham NL, Huvos AG, Marcove RC, Miller T, Shah IC. Radiation-induced sarcoma of bone. Cancer. 1971;28: 1087-99.

12. Min H, Hong M, Ma J, Zhang E, Zheng Q, Zhang J, et al. A new staging system for nasopharyngeal carcinoma in China. Int J Radiat Oncol Biol Phys. 1994;30:1037-42.

13. Tao CJ, Liu X, Tang LL, Mao YP, Chen L, Li WF, et al. Prognostic scoring system for locoregional control among the patients with nasopharyngeal carcinoma treated by intensitymodulated radiotherapy. Chin J Cancer. 2013;32:494-501.

14. Maleki S, Schlecht NF, Keller C, Diaz J, Moss J, Prystowsky $\mathrm{MB}$, et al. Lymphocytic host response to oral squamous cell carcinoma: an adaptive T-cell response at the tumor interface. Head Neck Pathol. 2011;5:117-22.

15. Almangush A, Bello IO, Keski-Santti H, Makinen LK, Kauppila JH, Pukkila M, et al. Depth of invasion, tumor budding, and worst pattern of invasion: prognostic indicators in earlystage oral tongue cancer. Head Neck. 2014;36:811-8.

16. Ueno H, Price AB, Wilkinson KH, Jass JR, Mochizuki H, Talbot IC. A new prognostic staging system for rectal cancer. Ann Surg. 2004;240:832-9.

17. Wang C, Huang H, Huang Z, Wang A, Chen X, Huang L, et al. Tumor budding correlates with poor prognosis and epithelial-mesenchymal transition in tongue squamous cell carcinoma. J Oral Pathol Med. 2011;40:545-51.

18. Gray RJ. A class of k-sample tests for comparing the cumulative incidence of a competing risk. Ann Stat. 1988;16:1141-54.

19. Wolden SL, Lamborn KR, Cleary SF, Tate DJ, Donaldson SS. Second cancers following pediatric Hodgkin's disease. J Clin Oncol. 1998;16:536-44.

20. Qin WJ, Luo W, Lin SR, Sun Y, Li FM, Liu XQ, et al. Sparing normal oral tissues with individual dental stent in radiotherapy for primary nasopharyngeal carcinoma patients. Ai 
Zheng. 2007;26:285-9.

21. Brandwein-Gensler M, Smith RV, Wang B, Penner C, Theilken A, Broughel D, et al. Validation of the histologic risk model in a new cohort of patients with head and neck squamous cell carcinoma. Am J Surg Pathol. 2010;34:676-88.

22. Xie N, Wang C, Liu X, Li R, Hou J, Chen X, et al. Tumor budding correlates with occult cervical lymph node metastasis and poor prognosis in clinical early-stage tongue squamous cell carcinoma. J Oral Pathol Med. 2015;44:266-72.

23. Paleri V, Wight RG, Silver CE, Haigentz M Jr, Takes RP, Bradley PJ, et al. Comorbidity in head and neck cancer: a critical appraisal and recommendations for practice. Oral Oncol. 2010;46:712-9.
24. Sanna G, Lorizzo K, Rotmensz N, Bagnardi V, Cinieri S, Colleoni $\mathrm{M}$, et al. Breast cancer in Hodgkin's disease and nonHodgkin's lymphoma survivors. Ann Oncol. 2007;18:288-92.

25. Ng AK, Bernardo MP, Weller E, Backstrand KH, Silver B, Marcus $\mathrm{KC}$, et al. Long-term survival and competing causes of death in patients with early-stage Hodgkin's disease treated at age 50 or younger. J Clin Oncol. 2002;20:2101-8.

26. Elkin EB, Klem ML, Gonzales AM, Ishill NM, Hodgson D, Ng $\mathrm{AK}$, et al. Characteristics and outcomes of breast cancer in women with and without a history of radiation for Hodgkin's lymphoma: a multi-institutional, matched cohort study. J Clin Oncol. 2011;29:2466-73. 